\title{
Intermediate Likelihood of Choledocholithiasis: Do All Need EUS or MRCP?
}

\author{
Nitin Jagtap ${ }^{1, \odot}$ Arun Karyampudi ${ }^{1}$ HS Yashavanth ${ }^{1}$ Mohan Ramchandani ${ }^{1}$ Sundeep Lakhtakia ${ }^{1, \odot}$ \\ Rakesh Kalapala ${ }^{1}$ Manu Tandan ${ }^{1}$ Jahangeer Basha ${ }^{1}$ Zaheer Nabi ${ }^{1} \quad$ Rajesh Gupta ${ }^{1}$ \\ DNageshwar Reddy ${ }^{1}$
}

${ }^{1}$ Department of Gastroenterology, Asian Institute of

Gastroenterology, Hyderabad, Telangana, India

Address for correspondence Nitin Jagtap, MD, DNB, Department of Gastroenterology, Asian Institute of Gastroenterology, Hyderabad, India (e-mail: docnits13@gmail.com).

J Digest Endosc:2021;12:19-23.

\section{Abstract \\ Keywords \\ - intermediate \\ likelihood \\ - choledocholithiasis \\ - predictors \\ - endoscopic ultrasound \\ - magnetic resonance cholangiopancrea- tography}

Background Recently updated guidelines for choledocholithiasis stratify suspected patients into high, intermediate, and low likelihood, with the aim to reduce risk of diagnostic endoscopic retrograde cholangiopancreatography. This approach has increased proportion of patients in intermediate likelihood making it heterogenous. We aim to substratify intermediate group so that diagnostic tests (endoscopic ultrasound/magnetic resonance cholangiopancreatography) are judicially used.

Methods This is a single-center retrospective analysis of prospectively maintained data. We used subset of patients who met intermediate likelihood of American Society of Gastrointestinal Endoscopy (ASGE) criteria from previously published data (PMID:32106321) as derivation cohort. Binominal logistic regression analysis was used to define independent predictors of choledocholithiasis. A composite score was derived by allotting 1 point for presence of each independent predictor. The diagnostic performance of a composite score of $\geq 1$ was evaluated in validation cohort.

Results A total of 678 (mean age [standard deviation]: 47.0 [15.9] years; 48.1\% men) and 162 (mean age 47.8 [14.8] years; 47.4\% men) patients in ASGE intermediate-likelihood group were included as derivation cohort and validation cohort, respectively. Binominal logistic regression analysis showed that male gender $(p=0.024$; odds ratio $[O R]=1.92)$, raised bilirubin $(p=0.001 ; \mathrm{OR}=2.40)$, and acute calculus cholecystitis ( $p=0.010$; OR $=2.04$ ) were independent predictors for choledocholithiasis. A composite score was derived by allotting 1 point for presence of independent predictors Using $\geq 1$ as cutoff, sensitivity and specificity for detection of choledocholithiasis were $80 \%$ (95\% confidence interval [CI]: $68.2-88.9$ ) and $36.2 \%$ (95\% Cl: $32.2-40.0$ ), respectively, in derivation cohort. Applying composite score in independent validation cohort showed sensitivity and specificity of $73.3 \%$ (95\% Cl: 44.9-92.2) and $40.1 \%$ (95\% Cl: 30.1-48.5), respectively.

Conclusion Substratification of intermediate-likelihood group of ASGE criteria is feasible. It may be useful in deciding in whom confirmatory tests should be performed with priority and in whom watchful waiting may be sufficient. published online December 5, 2020
DOI https://doi.org/ $10.1055 / \mathrm{s}-0041-1728233$ ISSN 0976-5042
(C)2020. Society of Gastrointestinal Endoscopy of India.

This is an open access article published by Thieme under the terms of the Creative Commons Attribution-NonDerivative-NonCommercial-License, permitting copying and reproduction so long as the original work is given appropriate credit. Contents may not be used for commercial purposes, or adapted, remixed, transformed or built upon. (https://creativecommons.org/licenses/by-nc-nd/4.0/). Thieme Medical and Scientific Publishers Pvt. Ltd. A-12, 2nd Floor, Sector 2, Noida-201301 UP, India 


\section{Introduction}

The American Society of Gastrointestinal Endoscopy (ASGE) has recently updated guidelines for management of choledocholithiasis. ${ }^{1}$ It provides stratified criteria for evaluation of suspected choledocholithiasis as: high likelihood, intermediate likelihood, and low likelihood. ${ }^{1}$ These guidelines appear robust in prediction of choledocholithiasis and more specific compared with previous guidelines. ${ }^{2-4}$

To consider a patient having "high-likelihood" criteria of common bile duct (CBD) stone, ASGE criteria includes: cholangitis, $\mathrm{CBD}$ stone on imaging or combination of total bilirubin $>4 \mathrm{mg} / \mathrm{dL}$, and dilated CBD on ultrasound (US). High-likelihood subjects can undergo either preoperative endoscopic retrograde cholangiopancreatography (ERCP) or intraoperative cholangiogram directly. On the contrary, patients with normal liver function tests (LFTs) and normal US are categorized to have "low likelihood" and can directly undergo cholecystectomy for symptomatic gallstones. The patients in between the two extremes or "intermediate likelihood" for choledocholithiasis (with abnormal LFTs and/or CBD dilatation on US or age $>55$ years) require further investigations like endoscopic ultrasound (EUS) or magnetic resonance cholangiopancreatography (MRCP) for confirmation of CBD stone. ${ }^{1}$

The intermediate-likelihood group appears heterogenous. Patients with symptomatic gallstones with mildly altered LFTs are required to undergo EUS or MRCP to rule out choledocholithiasis. Also, acute calculus cholecystitis (ACC) per se may be associated with altered LFTs without any choledocholithiasis.5.6 Earlier guidelines also considered acute biliary pancreatitis as "moderate predictor" of choledocholithiasis. ${ }^{4}$ Subsequent studies demonstrated that acute biliary pancreatitis is negative predictor for choledocholithiasis, as it is usually caused by sludge or microliths, which may pass out of papilla spontaneously. ${ }^{7}$ In our previous study, 9.6\% (65/678) ASGE intermediate-likelihood patients had choledocholithiasis. ${ }^{2}$ The newer guidelines aim to reduce the possibility of avoidable "diagnostic" ERCPs. There is also an apparent increase in number of patients in intermediate-likelihood group requiring to undergo EUS or MRCP. ${ }^{1,3,4}$ There is need to substratify patients in "intermediate-likelihood" group, so that diagnostic tests such as EUS and MRCP are used judiciously with better cost-benefit ratio.

In the present study, we aimed to evaluate and validate independent predictors for choledocholithiasis in patients with intermediate likelihood of choledocholithiasis as per ASGE criteria.

\section{Methods}

The Institutional Review Board approval was taken for this single-center retrospective study of prospectively maintained data. We included patients who underwent cholecystectomy for symptomatic cholelithiasis between January 2016 and December 2018. Those who had preoperative suspicion of choledocholithiasis and stratifying in intermediate likelihood of ASGE criteria were included in analysis as derivation cohort. This derivation cohort was derived from subset of patients from previously published study. ${ }^{2}$ An independent cohort of patients with intermediate likelihood of choledocholithiasis of ASGE criteria was included as validation cohort between May 2019 and October 2019. Patients with underlying liver disease, alcohol consumption, prior biliary surgery, prior ERCP, biliary strictures, portal cavernous cholangiopathy, and primary sclerosing cholangitis were excluded. Patients who were in high likelihood and low likelihood were excluded.

The data were entered in a standardized data collection sheet, which included demographic data such as age and gender, preprocedural LFTs, and US finding including presence or absence of CBD dilatation and presence of CBD stone and/or sludge. The CBD diameter of more than $6 \mathrm{~mm}$ on US abdomen was considered as dilated CBD. The clinical presentation of all included patients was stratified into ACC and acute biliary pancreatitis. Details of endoscopic procedures like EUS and ERCP and radiological procedure like MRCP were noted for confirmation of CBD stones. Patients in whom CBD stone was diagnosed within 6 months of follow-up after negative initial investigation were considered as missed CBD stones.

\section{Statistical Analysis}

The categorical and continuous data were mentioned as proportion and mean (standard deviation; SD), respectively. Confirmed CBD stone by ERCP or detected during 6-month follow-up was considered as dependent variable. For statistical analysis, serum bilirubin more than $1.8 \mathrm{mg} / \mathrm{dL}$, serum glutamic pyruvic transaminase more than three times ( $>120 \mathrm{U} / \mathrm{L})$, alkaline phosphatase more than upper limit of normal (>120 U/L), age, gender, presence of acute biliary pancreatitis, ACC, and dilated CBD on US were considered as independent variables. Univariate analysis followed by binominal logistic regression analysis using forward conditional method was done to ascertain independent predictors of CBD stones for the variables that are significant in univariate analysis. Odds ratio (OR) and confidence interval $(\mathrm{CI})$ were noted for statistically independent predictors. This predictor model was validated in validation cohort and diagnostic performance was calculated in terms of sensitivity, specificity, and positive and negative predictive values. A $p$-value of $<0.05$ was considered as statistically significant. Statistical analysis was done using SPSS version 23 (IBM Corp., Armonk, New York, United States) and MedCalc version 19.1.3 (MedCalc Software bv Ostend, Belgium).

\section{Results}

A total of 678 patients in ASGE intermediate-likelihood group were included as derivation cohort. The mean age (SD) was 47.0 (15.9) years and $48.1 \%$ were males. In the derivation cohort, 305 (45.0\%) had acute biliary pancreatitis, 169 (24.9\%) had ACC, and dilated CBD on US was seen in 230 (33.9\%) patients. An independent cohort of 162 patients was included as validation. The mean age (SD) was 47.8 (14.8) years and $47.4 \%$ were males. In validation cohort, 72 (44.4\%) had acute biliary pancreatitis, 40 (24.7\%) had ACC, and 60 
(37\%) had dilated CBD on US. The prevalence of choledocholithiasis in derivation cohort and validation cohort was $9.59 \%$ (65/678) and 9.26\% (15/162), respectively.

In univariate analysis in derivation cohort ( - Table 1), we found that male gender, ACC, dilated CBD in US, and serum bilirubin $>1.8 \mathrm{mg} / \mathrm{dL}$ were significantly associated with choledocholithiasis ( $p$-value $<0.05$ ). On multivariate analysis, using binominal logistic regression analysis using forward conditional method, we found that male gender $(p=0.024$; $\mathrm{OR}=1.92$ [95\% CI: $1.09-3.38])$, presence of ACC ( $p=0.010$; OR $=2.04$ [95\% CI: $1.18-3.53])$, and raised bilirubin $>1.8 \mathrm{mg} / \mathrm{dL}$ ( $p=0.001 ;$ OR $=2.40$ [95\% CI: $1.40-4.10]$ ) were independent predictors of choledocholithiasis. The composite score was calculated by allocating +1 score for each of the predictor, which ranges from 0 to 3 . Using $\geq 1$ as cutoff, sensitivity and specificity for detection of choledocholithiasis were $80 \%$ (95\% CI: 68.2-88.9) and 36.2\% (95\% CI: 32.2-40.0), respectively, in derivation cohort (-Table 2). Applying composite score in independent validation cohort showed sensitivity and specificity of 73.3\% (95\% CI: 44.9-92.2) and 40.1\% (95\% CI: 30.148.5), respectively.

\section{Discussion}

The current study evaluated independent predictors of choledocholithiasis in intermediate-likelihood group of ASGE guidelines. Male gender, presence of ACC, and raised bilirubin $(>1.8 \mathrm{mg} / \mathrm{dL})$ were independent predictors for choledocholithiasis in the patients with intermediate likelihood of choledocholithiasis. This evidence can be used for substratification of intermediate-likelihood patients to undergo confirmatory testing (EUS/MRCP). This substratification is validated in independent cohort with comparable sensitivity and specificity as that of derivation cohort.

There is an unmet need for substratification of intermediate-likelihood group for the following reasons. First, recent guidelines were aimed to reduce the risk of patients receiving avoidable diagnostic ERCP to reduce post-ERCP adverse events, ${ }^{1}$ due to which substantial proportion of patients within high-likelihood group as per previous guideline $^{4}$ are being included in intermediate likelihood. Second, as per ASGE diagnostic algorithms, 9.59\% patients have choledocholithiasis in intermediate-likelihood group. ${ }^{2}$ In low

Table 1 Univariable and multivariate analysis for ASGE “intermediate-likelihood group" for choledocholithiasis $(n=678)$

\begin{tabular}{|c|c|c|c|c|c|}
\hline \multirow[t]{2}{*}{ Variable } & \multicolumn{3}{|l|}{ Univariate } & \multicolumn{2}{|l|}{ Multivariate } \\
\hline & $\begin{array}{l}\text { CBD stone } \\
\text { present: } n(\%)\end{array}$ & $\begin{array}{l}\text { CBD stone } \\
\text { absent: } n(\%)\end{array}$ & $p$-Value & AOR (95\% CI) & $p$-Value \\
\hline \multicolumn{6}{|l|}{ Age } \\
\hline$<55 y$ & $37(8.7)$ & $387(91.3)$ & \multirow[t]{2}{*}{0.06} & & \\
\hline$>55 y$ & $28(11.0)$ & $226(89.0)$ & & & \\
\hline \multicolumn{6}{|l|}{ Gender } \\
\hline Male & $44(13.5)$ & $282(86.5)$ & \multirow[t]{2}{*}{0.001} & \multirow[t]{2}{*}{$1.92(1.09-3.38)$} & \multirow[t]{2}{*}{0.024} \\
\hline Female & $21(6.0)$ & $331(94)$ & & & \\
\hline \multicolumn{6}{|c|}{ Acute biliary pancreatitis } \\
\hline Yes & $23(7.5)$ & $282(92.5)$ & \multirow[t]{2}{*}{0.102} & & \\
\hline No & $42(11.3)$ & $331(88.7)$ & & & \\
\hline \multicolumn{6}{|c|}{ Acute calculus cholecystitis } \\
\hline Yes & $27(16.0)$ & $142(84)$ & \multirow[t]{2}{*}{0.001} & \multirow[t]{2}{*}{$2.04(1.18-3.53)$} & \multirow[t]{2}{*}{0.010} \\
\hline No & $38(7.5)$ & $471(92.5)$ & & & \\
\hline \multicolumn{6}{|c|}{ Dilated CBD on US } \\
\hline Yes & $13(5.7)$ & $217(94.3)$ & \multirow[t]{2}{*}{0.013} & & \\
\hline No & $52(11.6)$ & $396(88.4)$ & & & \\
\hline \multicolumn{6}{|c|}{ Bilirubin (>1.8 mg/dL) } \\
\hline No & $36(7.1)$ & $142(92.9)$ & \multirow[t]{2}{*}{0.004} & \multirow[t]{2}{*}{$2.40(1.40-4.10)$} & \multirow[t]{2}{*}{0.001} \\
\hline Yes & $29(17.0)$ & $471(83)$ & & & \\
\hline \multicolumn{6}{|c|}{ SGPT (>3x ULN) } \\
\hline No & $41(9.7)$ & $383(90.3)$ & \multirow[t]{2}{*}{0.925} & & \\
\hline Yes & $24(9.6)$ & $230(90.4)$ & & & \\
\hline \multicolumn{6}{|l|}{$\operatorname{ALP}(>\operatorname{ULN})$} \\
\hline No & $27(8.5)$ & 291 (91.5) & \multirow[t]{2}{*}{0.362} & & \\
\hline Yes & $38(9.6)$ & $322(90.4)$ & & & \\
\hline
\end{tabular}

Abbreviations: ALP, alkaline phosphatase; AOR, adjusted odds ratio; ASGE, American Society of Gastrointestinal Endoscopy; CBD, common bile duct; $\mathrm{Cl}$, confidence interval; SGPT, serum glutamic pyruvic transaminase; ULN, upper limit of normal; US, ultrasound. 
Table 2 Diagnostic performance of proposed substratification of ESGE and ASGE "intermediate-likelihood group" for choledocholithiasis

\begin{tabular}{|c|c|c|c|c|c|c|}
\hline & $\begin{array}{l}\text { True posi- } \\
\text { tive/Total } \\
\text { Positive }\end{array}$ & $\begin{array}{l}\text { True neg- } \\
\text { ative/Total } \\
\text { negative }\end{array}$ & $\begin{array}{l}\text { Sensitivity \% } \\
(95 \% \mathrm{CI})\end{array}$ & $\begin{array}{l}\text { Specificity \% } \\
(95 \% \mathrm{CI})\end{array}$ & $\begin{array}{l}\text { PPV \% } \\
(95 \% \mathrm{CI})\end{array}$ & $\begin{array}{l}\text { NPV \% } \\
(95 \% \mathrm{Cl})\end{array}$ \\
\hline \multicolumn{7}{|c|}{ ASGE intermediate-likelihood group composite score $\geq 1$} \\
\hline Derivation cohort $(N=678)$ & $52 / 444$ & $221 / 234$ & $\begin{array}{l}80 \\
(68.2-88.9)\end{array}$ & $\begin{array}{l}36.2 \\
(32.2-40.0)\end{array}$ & $\begin{array}{l}11.7 \\
(10.4-13.2)\end{array}$ & $\begin{array}{l}94.4 \\
(91.2-96.6)\end{array}$ \\
\hline $\begin{array}{l}\text { Validation cohort } \\
(N=162)\end{array}$ & $11 / 99$ & $59 / 63$ & $\begin{array}{l}73.3 \\
(44.9-92.2)\end{array}$ & $\begin{array}{l}40.1 \\
(30.1-48.5)\end{array}$ & $\begin{array}{l}11.1 \\
(8.2-14.9)\end{array}$ & $\begin{array}{l}93.7 \\
(86.2-97.2)\end{array}$ \\
\hline \multicolumn{7}{|c|}{ ASGE intermediate-likelihood group composite score $\geq 2$} \\
\hline Derivation cohort & $40 / 185$ & $468 / 493$ & $\begin{array}{l}61.54 \\
(48.64-73.35)\end{array}$ & $\begin{array}{l}76.35 \\
(72.78-79.66)\end{array}$ & $\begin{array}{l}21.62 \\
(17.84-25.95)\end{array}$ & $\begin{array}{l}94.93 \\
(93.21-96.23)\end{array}$ \\
\hline Validation cohort & $6 / 37$ & $116 / 125$ & $\begin{array}{l}40.00 \\
(16.34-67.71)\end{array}$ & $\begin{array}{l}78.91 \\
(71.42-85.20)\end{array}$ & $\begin{array}{l}16.22 \\
(8.81-27.93)\end{array}$ & $\begin{array}{l}92.80 \\
(89.42-95.16)\end{array}$ \\
\hline \multicolumn{7}{|c|}{ ASGE intermediate-likelihood group composite score = 3} \\
\hline Derivation cohort & $8 / 36$ & $57 / 641$ & $\begin{array}{l}12.31 \\
(5.47-22.82)\end{array}$ & $\begin{array}{l}95.27 \\
(93.28-96.81)\end{array}$ & $\begin{array}{l}21.62 \\
(11.63-36.63)\end{array}$ & $\begin{array}{l}91.11 \\
(90.33-89.83)\end{array}$ \\
\hline Validation cohort & $1 / 5$ & $143 / 157$ & $\begin{array}{l}6.67 \\
(0.17-31.95)\end{array}$ & $\begin{array}{l}97.28 \\
(93.18-99.25)\end{array}$ & $\begin{array}{l}20.00 \\
(2.90-67.69)\end{array}$ & $\begin{array}{l}91.08 \\
(83.01-93.28)\end{array}$ \\
\hline
\end{tabular}

Abbreviations: ASGE, American Society of Gastrointestinal Endoscopy; Cl, confidence interval; ESGE, European Society of Gastrointestinal Endoscopy; NPV, negative predictive value; PPV, positive predictive value.

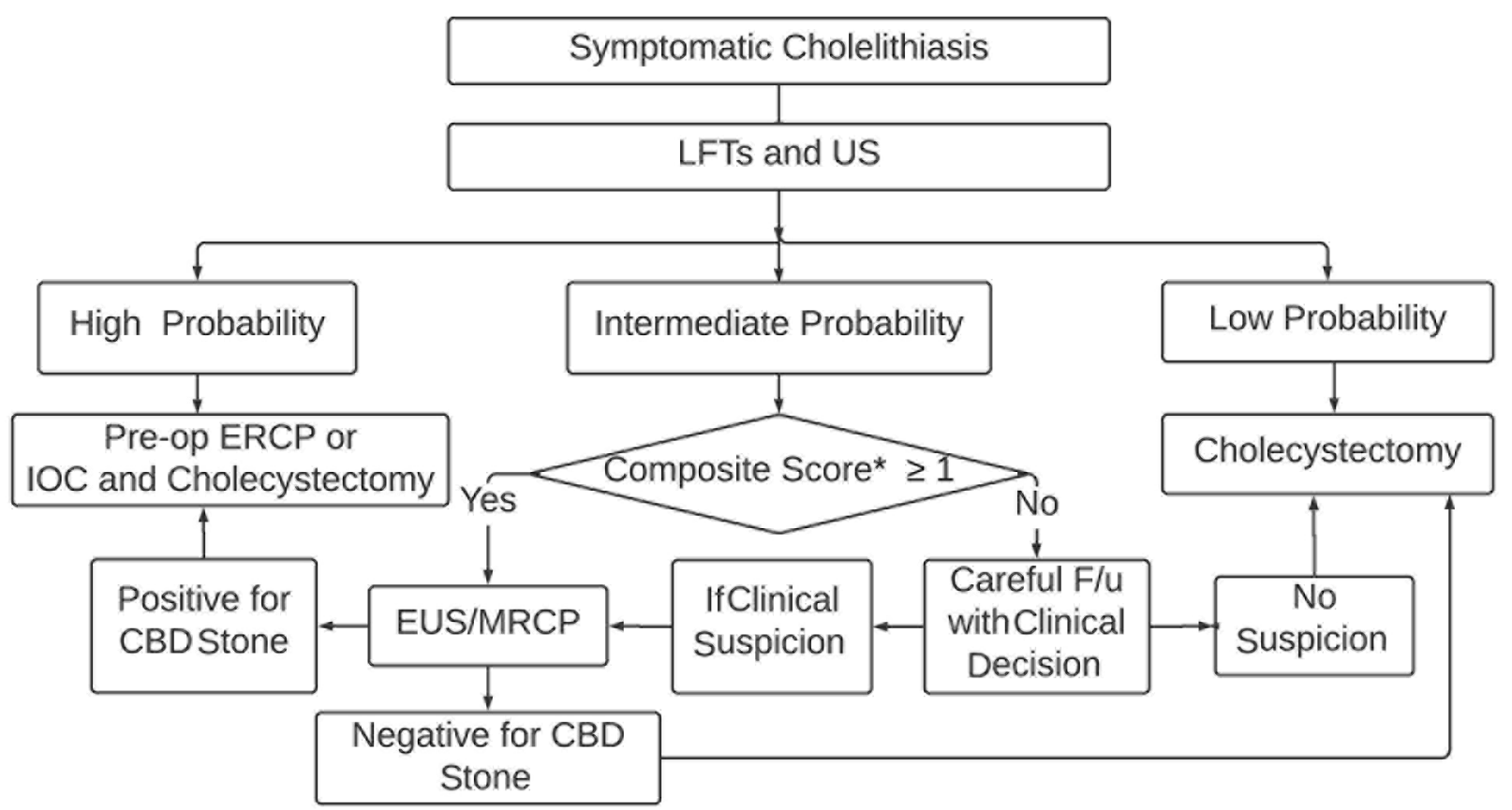

Fig. 1 Proposed modification of diagnostic algorithm for ASGE guidelines for choledocholithiasis. * Composite score derived from summation of positive predictors ( +1 for each). Abbreviations: ASGE, American Society of Gastrointestinal Endoscopy; LFTs, liver function tests; US, ultrasound (abdomen); Preop ERCP, preoperative endoscopic retrograde cholangiopancreatography; IOC, intraoperative cholangiogram; EUS, endoscopic ultrasound; MRCP, magnetic resonance cholangiopancreatography; CBD, common bile duct; F/u, follow-up.

likelihood, 3.7\% had choledocholithiasis, which means to find one additional patient with choledocholithiasis, 16.9 EUS or MRCP for ASGE are required. ${ }^{2}$ Third, acute biliary pancreatitis in previous guidelines were considered as moderate predictor of choledocholithiasis. ${ }^{4}$ But current guidelines have excluded it from diagnostic algorithms as acute biliary pancreatitis is a negative independent predictor for choledocholithiasis in recent studies. ${ }^{7}$ These patients tend to have tell-tale evidence of passed-out CBD stone such as thick-wall CBD or there may be CBD dilatation due to peripancreatic edema and/or fluid collection. ${ }^{8}$ Also, ACC can have alteration in LFTs with or without choledocholithiasis. ${ }^{6}$ Though confirmatory tests (EUS/MRCP) are safe and effective, it may not be cost-effective to perform them in all patients because of low pretest probability of choledocholithiasis in the intermediate group. ${ }^{9-11}$ 
We propose substratification of "intermediate-likelihood group" with patients having $\geq 1$ composite score, to improve utilization of EUS or MRCP in ASGE diagnostic algorithm. The proposed stratified algorithm is depicted in - Fig. $\mathbf{1}$. Composite score is calculated by allotting +1 point to each of positive predictor. In the ASGE intermediate-likelihood group, male gender, ACC, and serum bilirubin $>1.8 \mathrm{mg} / \mathrm{dL}$ were independent positive predictors. The composite score ranges from 0 to 3 . Those patients who do not meet this substratification can be closely followed and further decision for evaluation and management may be taken as per clinical judgment of the physician. A serial monitoring of LFTs to observe the trend may assist decision-making. Cases with no clinical suspicion may be considered for cholecystectomy directly. Conversely, in case of clinical suspicion, EUS or MRCP should be performed before cholecystectomy.

The proposed criteria are for substratification of patients within intermediate likelihood of choledocholithiasis, not to replace confirmatory tests such as EUS or MRCP. We chose composite score of $\geq 1$ as cutoff because of adequate sensitivity and specificity at this level. However, for composite score of $\geq 2$ or of 3 , there is drastic increase in specificity with similar decrease in sensitivity.

There are few limitations of the current study. In addition to retrospective analysis, study cohort consists of subset of patients from previously published paper. ${ }^{2}$ We did post hoc multivariable analysis to explore possibility of development of predictive model in the intermediate-likelihood group. We have validated this predictive model in the independent validation cohort, which showed comparable sensitivity and specificity to that of derivation cohort. Also, we did not study effect of serial LFT monitoring. Contrary to previous studies that showed contradictory results with serial monitoring for suspected choledocholithiasis in overall population, we believe serial LFT may have role in making clinical decision for subset of patients who have composite score less than $1.7,12,13$ Also, the prevalence of choledocholithiasis in the current study is around $10 \%$, which is at lower limit of expected prevalence of choledocholithiasis in patients within intermediate likelihood of choledocholithiasis. The probability of choledocholithiasis in intermediate likelihood is 10 to $50 \%$. Negative and positive predictive values are dependent on prevalence of condition, and sensitivity and specificity are prevalence independent.

In conclusion, substratification of the "intermediatelikelihood group" of ASGE risk stratification is suggested, which may be helpful to decide whether to perform confirmatory tests (EUS/MRCP) or wait and watch. ${ }^{14,15}$ However, these findings need to be externally validated in the prospective studies.

\section{Conflict of Interest}

None.

\section{References}

1 Buxbaum JL, Abbas Fehmi SM, Sultan S, et al. ASGE Standards of Practice Committee. ASGE guideline on the role of endoscopy in the evaluation and management of choledocholithiasis. Gastrointest Endosc 2019;89(6):1075-1105.e15

2 Jagtap N, Hs Y, Tandan M, et al. Clinical utility of ESGE and ASGE guidelines for prediction of suspected choledocholithiasis in patients undergoing cholecystectomy. Endoscopy 2020;52(7):569-573

3 Chandran A, Rashtak S, Patil P, et al. Comparing diagnostic accuracy of current practice guidelines in predicting choledocholithiasis: outcomes from a large healthcare system comprising both academic and community settings. Gastrointest Endosc 2020;:S0016-5107(20)34939-7

4 Maple JT, Ben-Menachem T, Anderson MA, et al. ASGE Standards of Practice Committee. The role of endoscopy in the evaluation of suspected choledocholithiasis. Gastrointest Endosc 2010;71(1):1-9

5 Chen $\mathrm{H}$, Jorissen $\mathrm{R}$, Walcott J, et al. Incidence and predictors of common bile duct stones in patients with acute cholecystitis: a systematic literature review and meta-analysis. ANZ J Surg 2019

6 Chisholm PR, Patel AH, Law RJ, et al. Preoperative predictors of choledocholithiasis in patients presenting with acute calculous cholecystitis. Gastrointest Endosc 2019;89(5):977-983.

7 He H, Tan C, Wu J, et al. Accuracy of ASGE high-risk criteria in evaluation of patients with suspected common bile duct stones. Gastrointest Endosc 2017;86(3):525-532

8 Tse F, Yuan Y. Early routine endoscopic retrograde cholangiopancreatography strategy versus early conservative management strategy in acute gallstone pancreatitis. Cochrane Database Syst Rev 2012;(5):CD009779).

9 Meeralam Y, Al-Shammari K, Yaghoobi M. Diagnostic accuracy of EUS compared with MRCP in detecting choledocholithiasis: a meta-analysis of diagnostic test accuracy in head-to-head studies. Gastrointest Endosc 2017;86(6):986-993

10 Chen YI, Martel M, Barkun AN. Choledocholithiasis: should EUS replace MRCP in patients at intermediate risk? Gastrointest Endosc 2017;86(6):994-996

11 Giljaca V, Gurusamy KS, Takwoingi Y, et al. Endoscopic ultrasound versus magnetic resonance cholangiopancreatography for common bile duct stones. Cochrane Database Syst Rev 2015; (2):CD011549

12 Rubin MI, Thosani NC, Tanikella R, Wolf DS, Fallon MB, Lukens FJ. Endoscopic retrograde cholangiopancreatography for suspected choledocholithiasis: testing the current guidelines. Dig Liver Dis 2013;45(9):744-749

13 Adams MA, Hosmer AE, Wamsteker EJ, et al. Predicting the likelihood of a persistent bile duct stone in patients with suspected choledocholithiasis: accuracy of existing guidelines and the impact of laboratory trends. Gastrointest Endosc 2015;82(1): 88-93

14 Adolfsson J. Watchful waiting and active surveillance: the current position. BJU Int 2008;102(1):10-14

15 Rawlinson J. The vanishing skill of watchful waiting. Br J Gen Pract 2017;67(656):108 